\title{
Single Brain Metastasis of Uterine Sarcoma Irradiated with Robotic Assisted LINAC-based Stereotactic Radiosurgery (Cyberknife): A Case Report
}

\author{
Selvi Tabak Dinçer ${ }^{1}$, (1D Emre Uysal1 ${ }^{1}$, (1) Selma Şengiz Erhan², (1) Ferdi Aksaray ${ }^{1}$ \\ 1 University of Health Sciences Turkey, Prof. Dr. Cemil Taş̧̧ığlu City Hospital, Clinic of Radiation Oncology, Istanbul, Turkey \\ 2 University of Health Sciences Turkey, Prof. Dr. Cemil Taşçığlu City Hospital, Clinic of Pathology, Istanbul, Turkey
}

\section{Abstract}

Brain metastasis in uterine leiomyosarcoma (ULMS) is very rare. Reported here is a 41-year-old woman with ULMS history who presented with multiple lung metastases, as she had undergone total abdominal hysterectomy, bilateral salpingo-oophorectomy, solitary lung metastasectomy, and 4 cycles of adjuvant chemotherapy. A multi-targeted tyrosine kinase inhibitor pazopanib was administered orally for recently developed multiple lung metastases. She has been followed for nearly 2 years without any treatment. With complaints of headaches, magnetic resonance imaging revealed a metastatic lesion in the vermis. The lesion was inaccessible, thus, neurosurgery could not be performed, and radiosurgery with Cyberknife was instead elected to be performed. She died due to massive hepatic and lung metastases after the brain radiosurgery completion.

Keywords: Uterine leiomyosarcoma, brain metastasis, radiosurgery, Cyberknife

\section{INTRODUCTION}

Uterine leiomyosarcoma (ULMS) is a rare tumor that represents $1-5 \%$ of all malignancies (1). ULMS is a highly aggressive tumor, thus, it frequently metastasizes to peritoneal cavity and omentum, lung, pelvic and paraaortic lymph nodes, and liver parenchyma (2). The incidence of brain metastasis is extremely rare $(2,3)$ and is strongly associated with lung metastasis $(1,4)$. We presented an uncommon case with brain metastasis from ULMS who was treated with stereotactic radiosurgery (SRS).

\section{CASE PRESENTATION}

In June 2014, a 41-year-old woman with a history of total abdominal hysterectomy (TAH) and bilateral salpingooophorectomy due to ULMS applied to our clinic. She had presented menorrhagia in September 2013, and uterine myomatosis was detected. She underwent TAH, and the histopathological diagnosis with leiomyosarcoma was made. The tumor diameter was $10 \mathrm{~cm}$ and mitosis was $>15 / 10$ high-power field (Figure 1). After the operation, thoracic and abdominopelvic computed tomographies (CTS) were performed for staging, and a solitary lung metastasis was found. Following pulmonary metastasectomy, 4 cycles of adjuvant chemotherapy with cisplatin and etoposide combination was performed. After 1 year, during her routine control, a $6 \times 8 \mathrm{~cm}$ mass was found in her left ovary, thus bilateral oophorectomy was performed, whereupon the pathology was reported as serous cystadenoma. In October 2014, CT of the thorax and abdomen revealed multiple metastatic lesions in the lung, with the largest one at $15 \mathrm{~mm}$ in diameter. Afterwards, oral treatment with a multi-targeted tyrosine kinase inhibitor pazopanib hydrochlorideat with daily doses of $800 \mathrm{mg}$ was initiated. Pancytopenia developed 
due to chemotherapy, and $48 \mathrm{MU}$ filgrastim SC (Neupogen $\left.{ }^{(R)}\right)$ was administered. Nausea and vomiting required antiemetic therapy. Control thoracic and abdominal CTs were performed

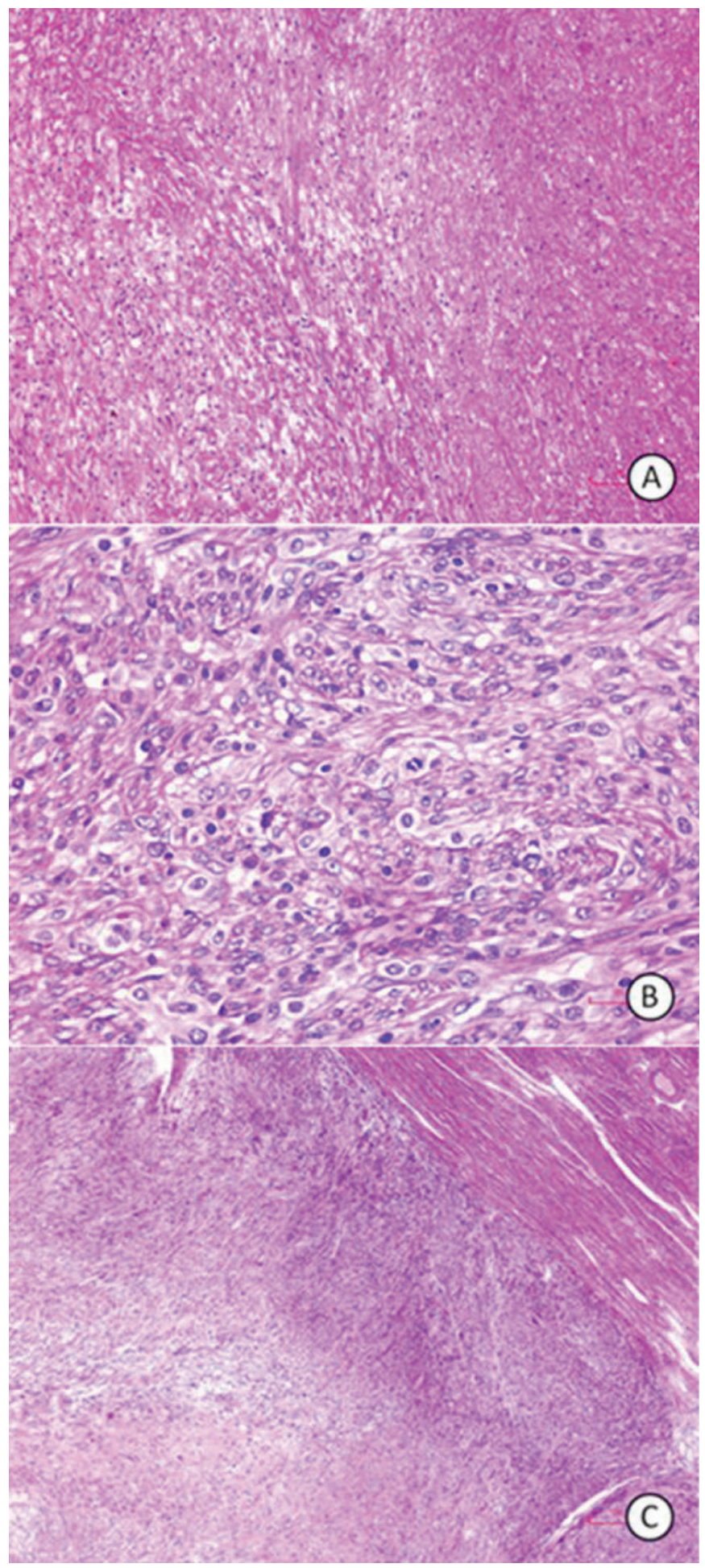

Figure 1. Histopathological examination of the uterus (H\&E section). (A) Cellular smooth muscle tumor with nuclear pleomorphism and area of coagulative necrosis. (B) Atypical mitoses. (C) Atypical cells beneath the fascicles of smooth muscle cells

H\&E: Hematoxylin and eosin every 3 months to evaluate the treatment response. Pazopanib was used continuously for 8 months until progression was seen in the lung metastasis on the thorax CT. Pazopanib was stopped in October 2015. She was followed-up for 20 months without any treatment. Then she visited the clinic due to headaches. Magnetic resonance imaging (MRI) revealed a $17 \mathrm{~mm}$ contrastenhancing metastatic lesion in the vermis (Figure 2). The lesion was inaccessible, thus, the patient underwent SRS with Cyberknife at a total dose of $22 \mathrm{~Gy}$ in single fraction instead of neurosurgery (Figure 3). Two weeks later she visited the emergency department with abdominal pain. Multiple liver metastases were detected on her abdominopelvic CT. She died 45 days after completion of radiation therapy because of progressive liver and lung lesions. Therefore, radiotherapy response could not be assessed with MRI.

\section{DISCUSSION}

ULMS is an aggressive tumor and surgery is the first treatment option for the control of primary disease; however, higher rates of recurrence and metastasis are present. Hematogenous and lymphatic metastases are frequently seen (5). Concomitant brain metastases are more frequently seen in patients with lung metastasis $(1,3)$.

In ULMS, where distant metastases are frequently seen, the effect of adjuvant chemotherapy on clinical outcomes is limited (6). Besides anthracycline and ifosfamide, novel agents such as gemcitabine, temozolamide, and taxanes are treatment options in locally advanced, recurrent, or metastatic disease. These cytostatic agents provide better systemic control and prolong overall survival (7). In a case study, pazopanib, a multi-targeted tyrosine kinase inhibitor with proven efficacy in soft tissue tumors, was used in a patient with pelvic lesions who had undergone resection of a cerebral metastatic lesion. Her pelvic lesions were kept under control for one year, without disease recurrence during this period (8). Brain metastases developing from gynecological malignancies, especially ULMS, are rarely seen; therefore, our literature information is limited to case series without robust guideline for optimal treatment for these patients (9).

The neurosurgical resection of the lesion is suggested as the mainstay treatment for brain metastasis of uterine sarcomas $(1,10)$. Wroński et al. (1) detected a mean survival of 7 months following surgical resection, whereas Bindal et al. (10) reported a median survival of 11.8 months after surgical resection in 21 patients with brain metastasis of uterine sarcoma. The decision of surgical treatment should be based on the extent of primary 
disease, location and number of brain metastases, time interval between initial diagnosis and development of brain metastasis, and expected life span of the patient.

In a prospective randomized study, Patchell et al. (11) reported that after surgical resection in the solitary brain metastases, postoperative whole brain radiotherapy (WBRT) achieved better disease control in the brain and decreased the number of deaths due to neurological etiologies, but did not contribute to survival.

No strong guideline was made for the management of brain metastasis of ULM; however, postoperative WBRT is frequently

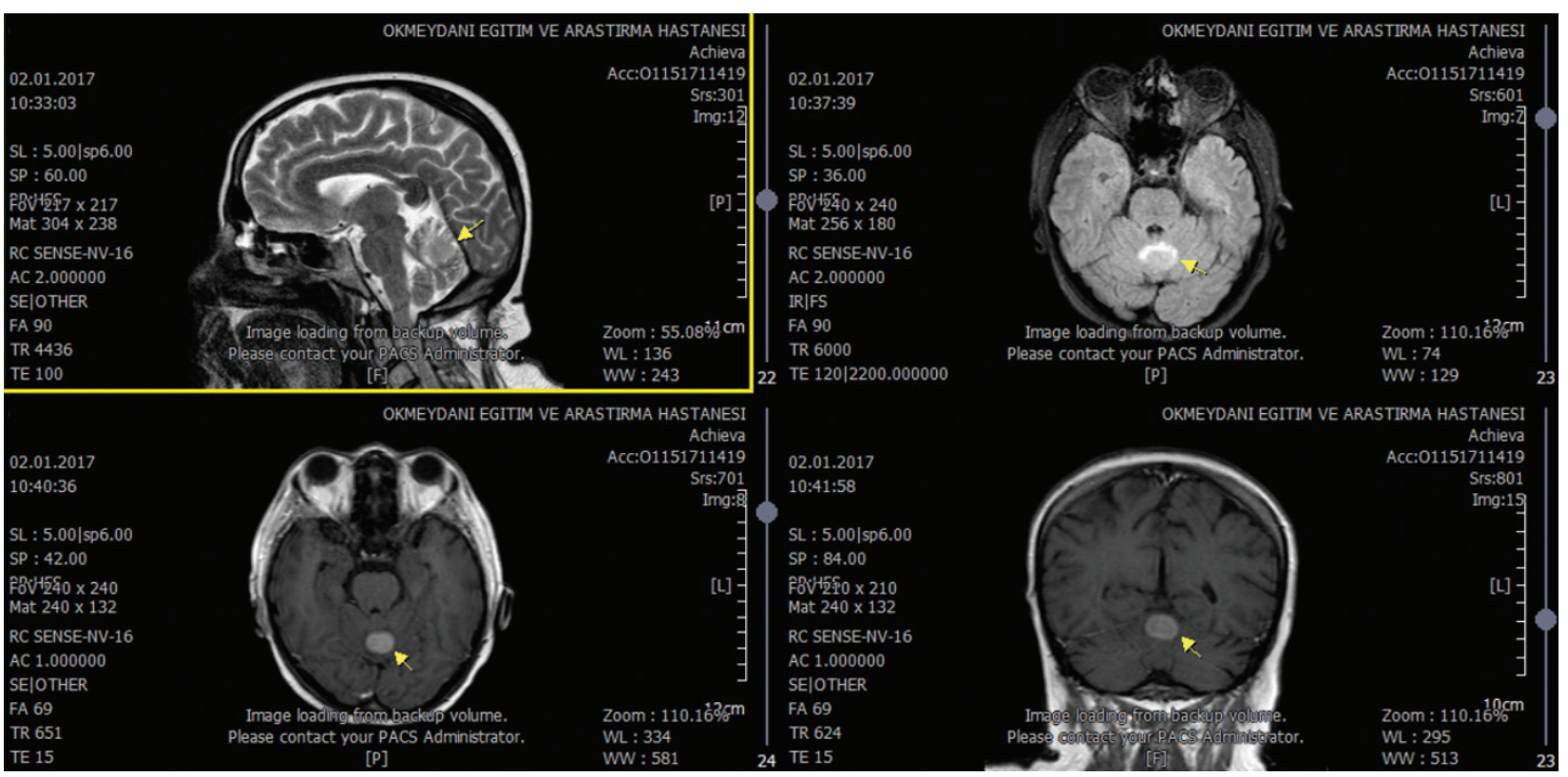

Figure 2. Initial MRI revealed a metastatic lesion in the vermis MRI: Magnetic resonance imaging

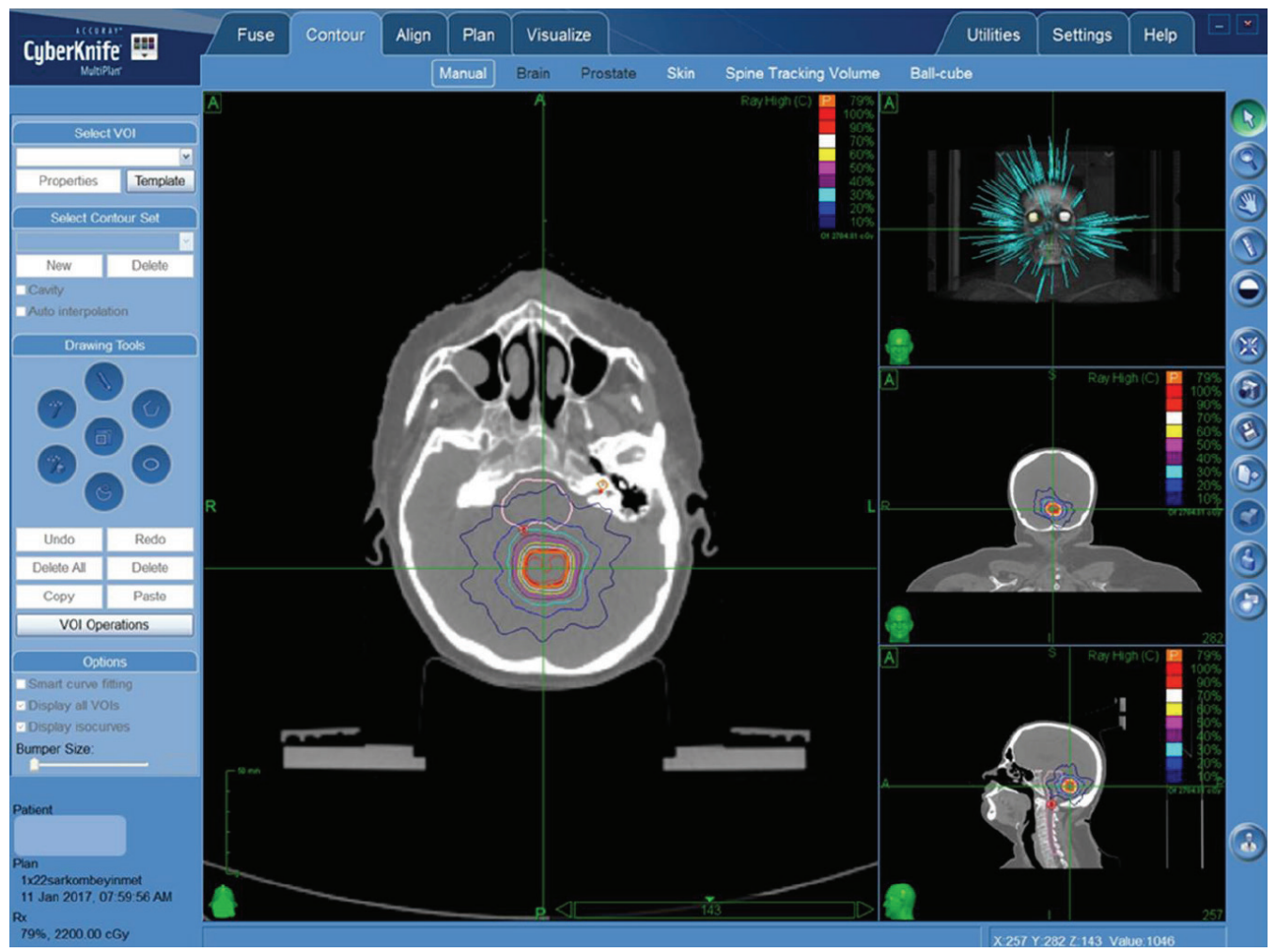

Figure 3. Representative of SRS treatment of metastasis in the vermis: Non-isocentric beam profile with dose distribution SRS: Stereotactic radiosurgery 
used as an adjuvant treatment. WBRT after neurosurgery can decrease the recurrence rates of brain metastasis, whereas long-term complications of radiotherapy increase. Therefore, neurocognitive decline should not be ignored in long-term survivals (12). However, WBRT can be preferred for widespread brain metastases and leptomeningeal invasion (13).

Nowadays, an increasing trend in the use of SRS for the management of oligometastatic brain tumors to avoid potential complications can be observed. Kasper et al. (13) performed SRS using Cyberknife for 20 brain metastases in 8 patients with gynecological malignancies and determined median distant brain progression-free survival and median overall survival times as 6 and 29 months, respectively. In retrospective studies of patients with gynecological malignancies who developed brain metastasis had undergone radiosurgery using Gamma Knife [(GKRS)-Elekta AB, Stockholm, Sweden], which is another radiotherapy method, excellent local control rates had been achieved without significant acute and long-term toxicities (14-16). In the study by Matsunaga et al. (14), median survival time was determined as 8 months, whereas more improved overall survival was detected in the group of patients with primary ovarian cancer and solitary brain metastasis whose extracranial metastases were under control. In addition, in the study by Shepard et al. (16), 16 patients were evaluated, brain metastases were detected in respective number of patients with ovarian cancer $(n=1)$, endometrial cancer $(n=6)$, cervical cancer $(n=1)$, and leiomyosarcoma $(n=1)$, and the median tumor dose of $20 \mathrm{~Gy}$ (range 10-22 Gy) had been delivered with GKRS. Local tumor control had been achieved in all patients. Median survival times following GKRS were 22.3 and 8.3 months in patients with primary ovarian cancer and endometrial cancer, respectively $(p=0.02)$. The patient with leiomyosarcoma died due to primary disease progression within 1 month after the treatment (16). Our patient died 45 days after radiotherapy completion as a result of liver and lung metastases progression.

SRS provides local control rates equivalent to those obtained from surgical series, and is effective in treating patients with surgically inaccessible tumors (17). Patients with limited brain disease, controlled systemic disease, and good performance status appear to benefit from therapy. Especially in these selected solitary brain metastatic lesions, SRS can be applied without WBRT in such a group of patients $(13,18)$.

The use of SRS alone was compared with SRS combined with WBRT in the group of patients with 1-3 brain metastases, and deterioration in neurocognitive functions was less frequently observed in the SRS arm at 3 months, which suggests that SRS is a preferred treatment approach for these patients (19). SRS was preferred for the case described here with solitary metastasis inaccessible for surgical resection located in the vermis.

\section{CONCLUSION}

In these patients, new systemic treatment options should be investigated in addition to radiosurgery to increase survival, as these patients are often lost due to extracranial tumor progression while maintaining local control of brain metastases.

\section{Ethics}

Informed Consent: Informed consent form was obtained before the patient was taken into treatment.

Peer-review: Externally peer-reviewed.

\section{Authorship Contributions}

Surgical and Medical Practices: S.T.D., F.A., Concept: S.T.D., E.U., F.A., Design: E.U., Data Collection or Processing: S.T.D., S.Ş.E., Analysis or Interpretation: S.T.D., Literature Search: S.T.D., S.Ş.E., Writing: S.T.D., E.U., F.A.

Conflict of Interest: No conflict of interest was declared by the authors.

Financial Disclosure: The authors declared that this study received no financial support.

\section{REFERENCES}

1. Wroński M, de Palma P, Arbit E. Leiomyosarcoma of the uterus metastatic to brain: a case report and a review of the literature. Gynecol Oncol 1994;54:237-41.

2. Rose PG, Piver MS, Tsukada Y, Lau T. Patterns of metastasis in uterine sarcoma. An autopsy study. Cancer 1989;63:935-8.

3. Yamada S, Yamada SM, Nakaguchi H, Murakami M, Hoya K, Matsuno A. A case of multiple brain metastases of uterine leiomyosarcoma with a literature review. Surg Oncol 2011;20:e127-31.

4. Abrahão CM, Maluf FC. Uterine leiomyosarcoma with central nervous system metastases. Ecancermedicalscience. 2015;5;9:515.

5. Honeybul S, Ha T. Leiomyosarcoma of the uterus metastatic to the brain: a case report. Arch Gynecol Obstet 2009;279:391-3.

6. Gadducci A, Landoni F, Sartori E, Zola P, Maggino T, Lissoni A, et al. Uterine leiomyosarcoma: analysis of treatment failures and survival. Gynecol Oncol 1996;62:25-32.

7. Vale CL, Tierney J, Bull SJ, Symonds PR. Chemotherapy for advanced, recurrent or metastatic endometrial carcinoma. Cochrane Database Syst Rev 201215;2012:CD003915.

8. Inoue $\mathrm{K}$, Tsubamoto $\mathrm{H}$, Tomogane $\mathrm{Y}$, Kamihigashi $\mathrm{M}$, Shibahara $\mathrm{H}$. Pazopanib-mediated long-term disease stabilization after resection of a uterine leiomyosarcoma metastasis to the brain: A case report. Gynecol Oncol Rep 2016;17:60-4. 
9. McMeekin DS, Kamelle SA, Vasilev SA, Tillmanns TD, Gould NS, Scribner $D R$, et al. Ovarian cancer metastatic to the brain: what is the optimal management? J Surg Oncol 2001;78:194-200; discussion 200-1.

10. Bindal RK, Sawaya RE, Leavens ME, Taylor SH, Guinee VF. Sarcoma metastatic to the brain: results of surgical treatment. Neurosurgery 1994;35:185-90; discussion 190-1.

11. Patchell RA, Tibbs PA, Regine WF, Dempsey RJ, Mohiuddin M, Kryscio RJ, et al. Postoperative radiotherapy in the treatment of single metastases to the brain: a randomized trial. JAMA 1998;280:1485-9.

12. Chang EL, Wefel JS, Hess KR, Allen PK, Lang FF, Kornguth DG, et al. Neurocognition in patients with brain metastases treated with radiosurgery or radiosurgery plus whole-brain irradiation: a randomised controlled trial. Lancet Oncol 2009;10:1037-44.

13. Kasper E, Ippen F, Wong E, Uhlmann E, Floyd S, Mahadevan A. Stereotactic radiosurgery for brain metastasis from gynecological malignancies. Oncol Lett 2017;13:1525-8.

14. Matsunaga S, Shuto T, Sato M. Gamma knife surgery for metastatic brain tumors from gynecologic cancer. World Neurosurg 2016;89:455-63.
15. Sadik ZHA, Beerepoot LV, Hanssens PEJ. Efficacy of gamma knife radiosurgery in brain metastases of primary gynecological tumors. J Neurooncol 2019;142:283-90.

16. Shepard MJ, Fezeu F, Lee CC, Sheehan JP. Gamma knife radiosurgery for the treatment of gynecologic malignancies metastasizing to the brain: clinical article. J Neurooncol 2014;120:515-22.

17. Alexander E 3rd, Moriarty TM, Davis RB, Wen PY, Fine HA, Black PM, et al. Stereotactic radiosurgery for the definitive, noninvasive treatment of brain metastases. J Natl Cancer Inst 1995;87:34-40.

18. Anupol N, Ghamande S, Odunsi K, Driscoll D, Lele S. Evaluation of prognostic factors and treatment modalities in ovarian cancer patients with brain metastases. Gynecol Oncol 2002;85:487-92.

19. Brown PD, Jaeckle K, Ballman KV, Farace E, Cerhan JH, Anderson SK, et al. Effect of Radiosurgery Alone vs Radiosurgery With Whole Brain Radiation Therapy on Cognitive Function in Patients With 1 to 3 Brain Metastases: A Randomized Clinical Trial. JAMA 2016;316:401-9. 